\title{
Vom Umgang mit Übertragung in der Mentalisierungsbasierten Therapie
}

\author{
Thomas Bolm (Utrecht)
}

Zusammenfassung: Patienten mitschweren strukturellen Entwicklungsstörungen, häufig mit einer Borderline-Persönlichkeitsstörung, haben eine hohe Übertragungsbereitschaft und stellen mit ihren heftigen Übertragungsphänomenen eine grosse Herausforderung für die Behandlungstechnik dar. Sie verfügen über noch keine ausgeprägte oder ausreichend stabile Mentalisierungsfähigkeit und können in der Regel nicht von klassischen Übertragungsdeutungen profitieren, welche die therapeutische Beziehung reflektieren und mit unbewussten Inhalten verknüpfen.

In diesem Beitrag geht es darum, wie die Mentalisierungsbasierte Therapie (MBT) durch ein stufenweises, prozessgerichtetes Vorgehen das Mentalisieren der Übertragung dennoch ermöglicht. Dies wird an einem Fallbeispiel verdeutlicht.

Schlüsselwörter: Borderline-Persönlichkeitsstörung, Bindung, Mentalisieren, Psychotherapie, Übertragung

1 Vom allgemeinen zum speziellen Fördern von Mentalisierung: Das Mentalisierungskonzept und die Mentalisierungsbasierte Therapie

\subsection{Mentalisieren als ein übergreifendes Thema aller Therapieschulen}

Das Mentalisierungskonzept ist ein weitgefasster theoretischer Ansatz mit breiter Anwendbarkeit (Bolm 2010a). Seit Beginn der Neunzigerjahre stellen Peter Fonagy und seine Arbeitsgruppe bindungstheoretische, entwicklungspsychologische, psychoanalytische und traumabezogene Erkenntnisse und Behandlungskonzepte auf den Prüfstand der empirischen Säuglings-, Kleinkind-, Psychotherapie- und neurobiologischen Forschung. Das Ergebnis ist eine Weiterentwicklung unseres Verständnisses von Mentalisierung, bzw. von Mentalisieren (Fonagy et al. 2002). Sie ist als Ergänzung zu bestehenden psychologischen Theorien gedacht und schulenübergreifend anwendbar. 
Beim Mentalisieren geht es um die - meist vorbewusste - mentale Fähigkeit,

a) die Aussen- und die Innenwelt eines Menschen,

b) Erlebnisse, Handlungen und Motivationen,

c) Intentionen und Resultate einer Handlung,

d) Körper, Psyche und Interaktion und

e) Einflüsse der Vergangenheit und gegenwärtiges Erleben und Verhalten

in einem bedeutungsvollen Zusammenhang miteinander erfahren und interpretieren zu können. Dies ist eine besonders wichtige Leistung des Ich im Zusammenhang mit dem Erleben von Stress. Wie später noch genauer ausgeführt wird, geht es bei bindungsgestörten Patienten vor allem um den Stress, der durch eine bindungsrelevante Beziehung und die damit verbundenen intensiven Emotionen entsteht.

Gute Mentalisierungsfähigkeit ist gekoppelt an die Bildung innerer Repräsentanzen dafür, dass für jeden Menschen jeweils ein persönliches Gefüge aus Erlebens- und Verhaltensweisen, Reaktionsmustern, Motivationen und Intentionen charakteristisch ist. Darum kann niemand objektiv oder lediglich von seiner eigenen Perspektive ausgehend wissen, wie das Erleben und die Motive des Anderen aussehen. Und darum sind dem Menschen der spielerische Umgang mit verschiedenen Perspektiven und das Bewusstsein für die Existenz von Irrtum, Täuschung und Übertragung möglich.

Die Mentalisierungstheorie macht empirisch untermauerte Aussagen darüber, wie sich die Mentalisierungsfähigkeit beim Menschen entwickelt, und von welchen Umwelteinflüssen dies abhängig ist. Vor allem ist dabei wichtig, dass Eltern ihrem Kind Präsenz und eine sichere Basis für sein Explorationsverhalten anbieten, das kindliche Erleben der Realität affektiv und inhaltlich auf ihr Kind abgestimmt spiegeln und die Intensität seiner Affekte modulieren. Damit können sie ihm dazu verhelfen, ein stabiles und kohärentes Selbst und eine auch in belastenden Situationen verfügbare Mentalisierungsfähigkeit zu entwickeln. Dies wird reflektierender Modus im Umgang mit der Realität genannt und ermöglicht im Erwachsenenalter, dass selbst bisher bewährte Erlebens- und Verhaltensmuster wenn nötig in Frage gestellt werden können und sich die Aufmerksamkeit auf Unbekanntes richten und das Fremde integriert werden kann (Bolm 2010b: 336).

Die Mentalisierungstheorie benennt drei hauptsächliche Modi, die auf ein noch wenig oder nur teilweise mentalisiertes Realitätserleben schliessen lassen. Sie sind in der Regel Durchgangsstadien der Mentalisierungsentwicklung. Oder sie treten im Erwachsenenalter sporadisch auf und werden dann, falls keine patholo- 
gische Fixierung vorliegt, durch Reparationsmechanismen aufgelöst. Es handelt sich um

a) den Äquivalenzmodus, in welchem Innenwelt und äussere Realität in ihrer Bedeutung noch nicht voneinander unterschieden und relativiert werden können,

b) den Als-ob-Modus, in dem Realitätsaspekte bzw. Selbstanteile zwar schon mit einer vom Konkreten losgelösten, abstrakten oder spielerischen Bedeutung versehen werden, aber unverbunden nebeneinander stehen oder zum Teil ausgeblendet werden, und

c) den teleologischen Modus, in dem sich die existentielle Angewiesenheit auf konkret wahrnehmbare Aktionen des Anderen zeigt. Die Intention hinter einer Handlung wird aus ihrem unmittelbar erfahrbaren Resultat erschlossen.

Auf die Dauer dysfunktionale, rigide Modi im Umgang mit der Realität entwickeln sich, wenn das Kind keine hinreichend sichere Umgebung erfährt, sei es durch traumatisierende äussere Lebensumstände oder durch mangelnde elterliche Mentalisierungsfähigkeit bedingt, oder durch beides. Das Erkennen des aktuellen Modus ist für die Auswahl passender Interventionen im Rahmen jeder Therapieschule eminent wichtig, besonders aber für den Umgang mit Übertragung, wie später ausführlich dargestellt werden wird.

\subsection{Mentalization-Based Treatment (MBT)}

Die Mentalisierungsbasierte Therapie ist eine spezielle Anwendung der Mentalisierungstheorie. Sie wurde von Bateman und Fonagy (Bateman und Fonagy 2004, 2006) für die störungsorientierte Behandlung schwerer und komplexer Persönlichkeitsstörungen entwickelt. Für die Behandlung von sehr schweren Borderline-Persönlichkeitsstörungen hat sich die seit 2004 manualisierte Methode in verschiedenen Settings als sehr erfolgreich, kosteneffektiv und gegenüber einer Standardtherapie oder einer übend-lösungsorientierten, gleichermassen intensiven Behandlung als überlegen herausgestellt (Bateman und Fonagy 1999, 2001, 2003, 2008, 2009). Die überlegene Effektivität der MBT wurde in zwei Settings bewiesen:

a) als maximal 18-monatige tagesklinische Behandlung, 5 Tage pro Woche, worauf eine gruppentherapeutische Nachsorge folgt

b) als ambulantes Intensivsetting über 18 Monate.

Es gibt jedoch auch Spielarten ambulanter Therapie mit 3-4 Jahren Behandlungsdauer (S. Karterud, mündliche Mitteilung). Das vormalige Team des Autors 
konnte in Deutschland mit einem dreimonatigen vollstationären Intensivsetting und einer einjährigen Nachsorgegruppe gute Erfahrungen machen (Bolm 2009b, Bolm und Herzog 2009).

Gemeinsamkeiten all dieser MBT-Angebote sind die Ausrichtung auf die schweren bis schwersten strukturellen Störungen und der kohärente, auf die Mentalisierungsförderung gerichtete Fokus. Dieser umfasst die neugierig-nichtwissende und antiregressive Haltung, aktive und flexible Interventionstechnik und Organisation der Therapie, das teamgestützte Arbeiten, die Einleitung mit einem psychoedukativ-übenden Pretreatment und die Kombination von Einzel- und Gruppentherapie sowie anderen Modulen.

MBT fokussiert explizit auf das Hier und Jetzt, die therapeutische Beziehung und die bewusstseinsnahen Inhalte. Die Vorgehensweise in Einzel- und Gruppengesprächen und nonverbalen Behandlungselementen ist psychodynamisch, also prozess-, beziehungs- und interaktionsorientiert unter Berücksichtigung der Übertragung und Gegenübertragung.

Nach dem anfänglichen Pretreatment mit Informationsvermittlung und expliziten Übungen ist MBT relativ arm an thematischen und strukturierenden Vorgaben. Dennoch gibt es eine übergreifende Strukturgebung in der MBT, nämlich die kohärente Ausrichtung aller an der Therapie beteiligten Personen und Therapiebestandteile auf die Suche nach gelungenem Mentalisieren, Mentalisierungsfehlern und deren Reparatur. Dies betrifft nicht nur die Patientenseite, sondern schliesst in bester psychodynamischer Tradition die gründliche Übertragungs-, Gegenübertragungs- und Organisationsanalyse mit ein.

Von einer Grundhaltung neugierigen Nichtwissens ausgehend haben die Interventionen vornehmlich klärenden Charakter und helfen dem Patienten, ein Bewusstsein für Mentales und Intersubjektivität zu entwickeln. Für diese Aufgabe muss der Therapeut gleichzeitig flexible therapeutische Rollenübernahme, äusserst beherzte strukturierende und regulierende Aktivität sowie Takt und Feingefühl zeigen. So sorgt er in einer Art dialektischem Prozess (Bolm 2010b) immer wieder für genug Sicherheitserleben zwischen ihm, dem Patienten und der Therapiegruppe. Dabei trägt MBT dem Umstand Rechnung, dass das Sicherheitserleben bei Patienten mit schweren Borderline-Störungen enorm fragil ist. Aus diesem Grund ist das tiefe Unbewusste nicht Gegenstand des gesprochenen Wortes, selbst wenn der MBT-Therapeut innerlich einer psychoanalytischen Wahrnehmungshaltung folgt. Gesprächsgegenstand ist lange das Bekannte, Bewusste, Offensichtliche, eine sichtbare Interaktion, Mimik, Gestik oder eine bewusste Kognition. Dies ist der Ausgangspunkt, von dem aus sich langsam auch unbewusstere Schichten der Psyche 
erschliessen, immer im Wechselspiel mit der Schaffung oder Wiederherstellung einer sicheren Basis im therapeutischen Kontakt.

Ziel der MBT ist nicht die Reinszenierung früherer Traumata in all ihrer Heftigkeit. Das Konflikterleben wird nicht gefördert, zum Beispiel in Form einer Übertragungsneurose, sondern aufgegriffen, wenn es spontan auftritt. Dem Erforschen - explizit nicht dem Deuten - spontaner Kommunikations- und Mentalisierungsbrüche und deren «Reparatur» wird der höchste Stellenwert eingeräumt. Meist geschieht das ganz unspektakulär, durch neugierige Fragen oder «mit leichter Hand» ausgeführte, «sich wundernde» Konfrontationen mit Realitätsaspekten. Manchmal muss der Therapeut jedoch seine ganze Energie aktiv in die Waagschale werfen, um den Explorationsprozess zu sichern.

Das Fonagy'sche Mentalisierungskonzept und die MBT haben sich eng aufeinander bezogen entwickelt. Dies geschah als britische Antwort einerseits auf die Herausforderungen, welche die Behandlung von schweren Borderlinestörungen mit sich bringen, andererseits auf die Grenzen bisheriger Methoden. Ihre Entstehung nahm keinen Bezug auf Entwicklungen von störungsorientierten Ansätzen für dieselbe Zielgruppe im nicht-englischsprachigen Raum, wie zum Beispiel die wesentlich früher entwickelte psychoanalytisch-interaktionelle Methode im Rahmen des Göttinger Modells (Lindner 2005, Streeck und Leichsenring 2009). So sind trotz einiger Unterschiede beträchtliche Überlappungen bei den Empfehlungen zu Grundhaltung und Behandlungstechnik entstanden. Zu ihnen gehören der Verzicht auf (Übertragungs-) Deutungen und die selektive Authentizität bei der Mitteilung der Gegenübertragung.

2 Warum der Inhalt von Übertragungsdeutungen in Zuständen schwerer Mentalisierungsstörung nicht genutzt werden kann

\subsection{Die Auswirkung von situativem und chronischem Stress auf die Fähigkeit zum Mentalisieren der Übertragung}

Aus vielen Borderline-Behandlungen ist bekannt, dass heftige negative (Teilobjekt-) Übertragungen sich häufig aus kindlichen Erfahrungen herleiten, dass bindungsrelevante und nahe Beziehungen mit Verlust, Willkür, Gewalt oder psychischem bzw. sexuellem Missbrauch gekoppelt sind. Oder es gab vor allem Mangel und Vernachlässigung. Es ging in vielen Biographien dieser Patienten darum, sich in unüberschaubar komplexen oder kaum erträglichen Situationen dennoch zurecht zu finden und zugleich die kognitive und emotionale Tragweite der Traumatisierungen von sich fernzuhalten. Eine Folge davon ist das schnell 
anspringende Alarmsystem, durch das Flucht- und Kampf-assoziierte Selbst- und Objektrepräsentanzen wieder aktiviert werden.

Die hohe Übertragungsbereitschaft der Borderline-Patienten führt jedoch nicht nur zur niedrigschwelligen Wiederbelebung archaischer Objektbeziehungsqualitäten, sondern triggert die bei diesen Patienten hyperaktiven physiologischen Angst-Stress-Systeme. Bereits der spontaneVerlauf eines therapeutischen Prozesses ist hierfür Auslöser genug. Wenn nämlich der Therapeut zur bedeutungsvollen Person wird, dann werden bei einem Patienten mit einer schweren (bindungs-) traumatisierenden Beziehungserfahrung durch heftige Emotionen und Stress diejenigen Regionen des Frontalhirns gehemmt, die für das Mentalisieren verantwortlich sind (Nolte et al. 2010). In solchen Zuständen können komplexe Interventionen, wie zum Beispiel Übertragungsdeutungen, durch den Patienten nicht mehr entschlüsselt werden, weil ihm durch die momentane, stressbedingte Mentalisierungsstörung seine intellektuellen, reflektierenden und empathischen Fähigkeiten nicht mehr zur Verfügung stehen.

Doch die Störung reflektiven Verhaltens ist weitreichender, weil in der Kindheit dieser Patienten keine repräsentationale Basis für ein mentalisierendes Verständnis stressvoller Interaktionen gelegt ist, eine Basis, die modulierend und regulierend auf die Affekte einwirken könnte: Die Patienten funktionieren in angespannten Zuständen im Äquivalenzmodus, d.h. sie können keinen Unterschied machen zwischen der Bedeutung, die ihre inneren Vorgänge, zum Beispiel Fantasien oder Erinnerungen, für sie haben, und dem was ein anderer denkt, beabsichtigt und tut. Sie werden vielleicht schon das Angebot einer von der ihren abweichenden Interpretation als Indoktrinationsversuch ansehen und in ihr inneres Bedrohungsszenario einbauen. Oder sie werden es im Als-ob-Modus akzeptieren, der angebotenen Deutung bewundernd zustimmen und dennoch in keinen inneren Auseinandersetzungsprozess kommen.

\section{$2.2 \quad$ Fallbeispiel (Teil 1)}

Die 36-jährige Frau M. besucht seit 4 Monaten meine MBT-Abteilung für ausnahmslos schwere bis schwerste Borderline-Störungen, nachdem sie zahlreiche andere Therapieanläufe nach mehr oder weniger kurzer Zeit abgebrochen hat. Sie kommt nach einem ihrer zahlreichen Suizidversuche in einer Situation, in der ihre Kompensationsstrategien, vor allem beruflicher Erfolg und Aufopferung für Freunde und Familienmitglieder, nicht mehr funktionieren und zu einer totalen Entkräftung und Verstrickung in berufliche und familiäre Konflikte geführt haben. 
Die Patientin leidet seit 15 Jahren unter massiven Depressionen mit chronischen Suizidgedanken, einer gemischten Angststörung mit generalisierter Angst, Panikattacken und Agoraphobie sowie einer dissoziativen Symptomatik mit Depersonalisations- und Derealisationsphänomenen. In diesen Episoden begeht sie Drogen- und Alkoholexzesse und unternimmt Suizidversuche mit Tabletten. Regelmässig fügt sie sich Schnitte an Armen und Beinen zu.

Aus struktureller Sicht wechseln sich eher auf ein neurotisches Niveau hinweisende Abwehrmechanismen, wie zum Beispiel Reaktionsbildung und Rationalisierung, mit dem Zusammenbruch der Realitätsprüfung und psychosenahen paranoiden Befürchtungen sowie intellektualisierendem Psychologisieren ab. In den psychosenahen Phasen zeigt sich eine sehr niedrige Übertragungsschwelle für die Auslösung posttraumatischer Reaktionsmuster und traumabezogener Kognitionen.

Die Patientin wuchs in den Niederlanden in einer Familie nordafrikanischer Herkunft auf. In dieser war sie massiver physischer Gewalt und verbaler Erniedrigung von Seiten des Vaters und der Brüder ausgesetzt. Von einem entfernteren Verwandten wurde sie mehrfach sexuell missbraucht, traute sich jedoch nicht, sich jemandem anzuvertrauen. In ihrer Familie lernte sie, ihr Daseinsrecht aus der selbstverleugnenden und sehr verausgabenden Hilfe für ihre Familienangehörigen zu ziehen. Eine stabile Partnerschaft oder Familie hat sie nicht. Ihre Karriere im Dienstleistungsbereich erleidet stets Brüche, wenn sie in Konflikte mit anderen oder zwischen anderen gerät. Dann steht sie völlig neben sich, kann nicht mehr denken oder sprechen, oder sie wird zur gnadenlosen Furie, die ohne jede Verhältnismässigkeit andere in Grund und Boden kritisiert. Dies kann sie im Nachhinein nicht relativieren, sondern versandet in sehr eloquenten cotherapeutischen Monologen. Damit ruft sie vor allem zwei Reaktionsmuster bei anderen hervor, zum einen, dass Hilfsbedürftige bei ihr Schutz und Trost suchen, zum anderen Ärger über ihren unangemessenen und wenig authentischen Umgang mit Konflikten, Aggression und Selbstfürsorge.

Frau M. besucht eines der 4 in meiner Klinik angebotenen MBT-Settings, das «Intensive Outpatient Program» (IOP). Nach Abschluss der initialen psychoedukativen und übenden Phase begann sie vor nunmehr 6 Wochen den zweiten Behandlungsabschnitt, die intensive einzel- und gruppenpsychotherapeutische Komplexbehandlung. Ich bin einer ihrer beiden Gruppenpsychotherapeuten, während sie mit meiner Cotherapeutin ihre Einzelgespräche führt. Weil diese Gruppe ausnahmsweise nicht slow-open geführt wird sondern gemeinsam im geschlos- 
senen Gruppenverband startete, befindet sich der gesamte Gruppenprozess noch in der Anfangsphase.

MBT-(Gruppen-)Therapeuten vermeiden lange Schweigepausen, fragen aktiv nach und fokussieren, regulieren aktiv das Anspannungsniveau, knüpfen aktiv Verbindungen zwischen den Äusserungen der Teilnehmer und nehmen mit ihren Ich-Funktionen und dem selektiven Einbringen ihrer Gegenübertragung eine aktive Mitspielerrolle ein. All dies ist erforderlich bei einer Gruppenzusammensetzung mit ausnahmslos schwer bis schwerst strukturell gestörten Patienten.

Frau M. verfällt auch in dieser antiregressiven Umgebung in dissoziatives dumpfes, abwesendes Schweigen ohne Blickkontakt oder in eloquente, psychologisierende Monologe. Wenn sie dissoziiert, möchte sie nicht angesprochen werden, sichert aber zu, sich später wieder ins Gruppengespräch einzuschalten. Eine Verbindung zwischen ihrer Reaktion und möglichen aktuellen Auslösern kann sie nicht benennen, wiederholt nach solchen Vorfällen aber stereotyp paranoide Beschuldigungen gegen die anderen Gruppenmitglieder. Wenn diese daraufhin Frau M. mit Interpretationsversuchen helfen wollen, behandelt sie deren Inhalt und Form erschöpfend psychologisierend, ohne jedoch in einen inneren Auseinandersetzungsprozess zu kommen. Später, ausserhalb der Gruppensitzungen gibt sie vernichtende Kommentare ab. Wenn Gruppenmitglieder ihr ihren Ärger über dieses Verhalten mitteilen, verfällt sie in hilfloses Schweigen. Nach solchen Episoden ist sie in Gefahr, sich selbst auf verschiedene Weise aktiv körperlich oder sozial zu schädigen. Statt zu versuchen, darüber im Nachhinein zu reflektieren, ist sie schnell wieder die zweite «Cotherapeutin» der Gruppe.

Deutlich kommt zu Tage, dass sie innerlich nicht auf ein Konzept von Intersubjektivität zurückgreifen kann, um verschiedene Perspektiven anzuerkennen, zu nutzen oder vorwegzunehmen, und dass sie weder die eigene noch eine von der ihren abweichende Interpretation der Realität einem hinreichenden Mentalisierungsprozess unterzieht. Umso ungefilterter und heftiger erlebt sie die archaischen Gefühlsqualitäten, die durch Übertragungsauslöser für traumatische Erlebensmuster hervorgerufen werden.

\section{Wie geht die MBT mit Übertragungsphänomenen um?}

\subsection{Durcharbeiten der Übertragung - aber mentalisierungsorientiert}

«Übertragung wird im allgemeinen definiert als das Erleben von Gefühlen, Phantasien und Abwehrhaltungen und letztlich von Beziehungsrepräsentanzen, in die die mehr oder weniger phantasmatische Verarbeitung früherer, traumatisierend 
und konflikthaft erlebter Interaktionsprozesse mit wichtigen Bezugspersonen des Kindes eingegangen ist. Dieses Erleben, das dieWahrnehmung, die Aufmerksamkeit, das Erinnern, die Art und Weise zu sprechen, aber auch die Körpersprache des Patienten beeinflusst, tritt in der jetzigen Therapiesituation gegenüber dem Therapeuten auf, [...] der, anders als in alltäglichen Interaktionssituationen, idealiter einen analytischen Raum für dieses Erleben bereitstellt» (Mertens 1993: 186).

Diesem psychoanalytischen Modell folgt die MBT, wenn sie Übertragung als ein reales Geschehen im Hier und Jetzt der therapeutischen Interaktion ansieht, das sowohl von gegenwärtigen wie vergangenen Einflüssen beeinflusst wird, und dem in der Therapie ein korrespondierender «Mentalisierungsraum» zur Seite gestellt wird. Berücksichtigt werden die besonderen intrapsychischen Entwicklungsprozesse und behandlungstechnischen Notwendigkeiten, um dies auch bei schweren Borderline-Störungen zu realisieren. Insbesondere gilt die Aufmerksamkeit denjenigen Prozessen, welche die Qualität und Sichtweise der therapeutischen Beziehung viel basaler beeinflussen als auf dem Niveau phantasmatischer Beeinflussung. Das Ziel der MBT ist die Mentalisierungsförderung, also die innerlich verankerte Anerkennung der Relevanz von persönlichen Gefühlen, vielfältigen Einflüssen aus Vergangenheit und Gegenwart und einer Menge Vermutungen und Phantasien übereinander und über sich selbst. Mentalisieren ist sicher nicht ein pseudoobjektives «Wissen» im Sinne von «Er ist einfach ein böser Mensch!», «Alle Männer sind Schweine!», «Ich bin Borderline!». Deshalb wäre es übrigens auch eine Mentalisierungsbremse, wenn einer nachdrücklichen Projektion eine ebenso nachdrücklich objektiv gemeinte Interpretation des Therapeuten entgegengesetzt würde.

Zu den notwendigen Voraussetzungen für das Gelingen der Übertragungsarbeit in der MBT gehört eine hohe persönliche Präsenz des Therapeuten, der die bindungs-, rahmen-, prozesssichernden und -regulierenden Ich-Funktionen in der Behandlung repräsentiert. Das kann er mit seiner Präsenz selbst dann tun, wenn Inhalte von Therapieabsprachen und Verträgen dem Patienten in einem hoch angespannten oder dissoziativen Zustand mit stark eingeschränkter Mentalisierungsfähigkeit nicht mehr verfügbar sind. Ein passiv abwartender, persönlich unkenntlicher Therapeut dagegen könnte für den Patienten zwar zum Übertragungsauslöser für ein abwesendes, vernachlässigendes Teilobjekt werden, aber dann nicht mehr für den notwendigen analytischen Raum sorgen. Anders als in der psychodynamischen Behandlung von Patienten mit neurotischem Strukturniveau ist also für dieses Klientel persönliche Präsenz kein 
Hindernis sondern eine Notwendigkeit für das Erforschen und Bearbeiten von Übertragungsmustern.

Aktiv und schnell greift der MBT-Therapeut aktuelle Lebensprobleme und Konflikte des Patienten auf, die sich in der therapeutischen Beziehung und ausserhalb dieser ereignen, exploriert sie und fördert den mentalisierenden Umgang mit ihnen. Weder bezieht der Therapeut im Gespräch alle Zeichen für Übertragungsphänomene stereotyp auf sich noch lenkt er die Aufmerksamkeit auf das Dort und Damals.

\subsection{Vorbereitung aufdas Mentalisieren der Übertragung}

MBT schlägt eine abgestufte Strategie von übertragungsbezogenen Interventionen vor, die dafür sorgen, dass die Mentalisierungsförderung nicht blockiert wird (Bateman und Fonagy 2004: 207 ff., Bolm 2009b: 107 ff.). Zu einer Blockade würde es bei zu hoher kognitiver und emotionaler Komplexität und bei zu stressvoller affektiver Beteiligung kommen.

MBT bereitet den Patienten explizit auf die Arbeit an der Übertragung vor. Dies geschieht durch

a) Informationsvermittlung über das Übertragungskonzept,

b) die gemeinsame Formulierung eines dynamischen Fokus, der Symptome, dysfunktionale Interaktion, pathologische mentale Vorgänge und Ressourcen in einen mentalisierungsbezogenen Zusammenhang bringt, und

c) die explizite Benennung von «Übertragungsmarkern», womit Kennzeichen für bestimmte dominante Übertragungsmuster gemeint sind. Ausdrücklich wird auf das wahrscheinliche Wiederauftreten dieser Muster gegenüber den Mitpatienten, der Gruppe, dem Therapeuten oder Aussenstehenden hingewiesen. Für einen solchen Fall sind die Übertragungsmarker die Signale für eine ausführliche Exploration der Situation.

\section{Fallbeispiel (Teil 2)}

Bei Frau M. kommt zu Tage, dass sie sich öfter aus einem Kontakt zurückzieht (Übertragungsmarker 1) oder andere entwertet und «auflaufen» lässt (Übertragungsmarker 2), wenn sie sich selbst zurückgesetzt und entwertet fühlt, so wie in ihrem Elternhaus durch ihren Vater. Der habe nur Augen und Ohren für ihre Brüder gehabt, selbst als sie ihn nach den sexuellen Übergriffen durch den entfernten Verwandten am meisten gebraucht hätte. Auf ihre Mutter habe sie auch nicht zählen können, die habe mit sich selbst genug zu tun gehabt. Dies alles weiss 
sie in entspannten Momenten, aber das hilft ihr nicht in Stresssituationen. Wenn sie die beschriebenen Erlebnisqualitäten im Kontext einer für sie wichtigen Beziehung erlebt, wie z. B. mit einem potentiellen Partner, einem Vorgesetzten oder einem Freund, von dem sie sich abhängig fühlt, bekommt ihr Erleben eine traumatische Qualität. Die Folge ist, dass sie schon, wenn jemand beginnt, ihr wichtig zu werden, dissoziative Zustände bekommt (Übertragungsmarker 3).

\subsection{Schritte zur Mentalisierung der Übertragung}

Wenn es in der MBT zu relevanten Übertragungsvorgängen kommt, beginnt die mentalisierungsfördernde Arbeit mit der Klärung und Benennung des vorrangigen Affektes und des Kontextes im Hier und Jetzt. Weil dem Patienten in einem solchen Moment keine Repräsentanz für Irrtum oder Intersubjektivität zurVerfügung steht, kann er seine momentane Erlebensrealität nicht hinterfragen. Deshalb stellt sich der Therapeut empathisch an die Seite des Patienten, weist die Projektion nicht zurück und deutet sie auch nicht, sondern exploriert das Entstehen dieser Sichtweise und validiert sie. Dadurch behält der Patient zunächst seine Entlastungsmöglichkeit durch Externalisierung. Diese Vorgehensweise entlastet den Patienten oft deutlich von inneren Spannungen und Ängsten vor Kohärenzverlust.

Übertragungsmuster werden erst dann vorsichtig in Frage gestellt, wenn genügend mentalisierende Kapazität für das Einnehmen unterschiedlicher Perspektiven erarbeitet ist. Wenn der Patient anerkennen kann, dass seine Sichtweise nur eine unter vielen möglichen und an seine individuellen Erlebensmuster gebunden ist, nennt man dies Mentalisieren der Übertragung. Statt also an den «richtigen» oder «realistischen» Inhalten der Realitätssicht zu arbeiten, ist das Ziel der MBT, den mentalisierenden Prozess und den dementsprechenden Repräsentanzenaufbau zu fördern. Erst wesentlich später sollten Übertragungsdeutungen gebraucht werden.

\section{Fallbeispiel (Teil 3)}

Frau M. will seit einer Woche nicht mit mir reden und vermeidet jeden Blickkontakt mit mir. Dieses Verhalten zeigt nicht die bisherige dissoziativ bedingte Generalisierung, von der alle Gruppenteilnehmer gleichermassen betroffen sind, sondern zielt direkt auf mich. Ihr vordergründig gesehen unvermittelt in einer Gruppensitzung geäusserter Vorwurf, dass ich nicht mit ihr arbeiten wolle, was es dann noch solle, dass ich sie nach ihrer Meinung frage, trifft mich. Zum einen empfinde ich es im Grossen und Ganzen anders, zum anderen fühle ich mich auch ertappt, denn ich finde ihr dissoziatives Schweigen, aber mehr noch ihr 
Psychologisieren anstrengend und ärgere mich zunehmend darüber. Ich verlege den folgenden Dialog aus didaktischen Gründen vom Gruppenkontext in das Setting eines Einzelgesprächs, um nicht noch die Mehrpersonendimension hinzufügen zu müssen. Darüber ist an anderer Stelle ausführlich geschrieben worden (Bolm 2009a, Bolm 2009b: 119 ff.).

T.: Wenn ich Ihre heftige Kritik so höre, dann muss ich sagen, sie trifft mich und überrascht mich auch. So laut und scharf haben Sie mich noch nie vorher angesprochen. Wie geht's Ihnen denn innerlich damit, dass ich so unwillig wirke?

(M. schweigt, sieht mich mit Stirnfalten und zusammen gepressten Lippen an)

T.: Das ist wohl kein sehr angenehmer Zustand, in dem Sie sich befinden, oder?

M.: Jaaah, ich bin echt schlecht drauf, was denken Sie denn!

T.: Und was meinen Sie genau mit «schlecht drauf»? Ich kann mir darunter sehr viel Verschiedenes vorstellen... .

M.: Was soll denn das Gefrage schon wieder. Ich bin wütend auf Sie, ja. Denn Sie nehmen mich nicht ernst, und Sie wollen sowieso nicht, dass ich hier in der Gruppe sitze.

T.: Oh! Ich dachte bisher, dass ich ernsthaft mit ihnen arbeiten will. Aber wenn Sie so einen anderen Eindruck haben, dann sollten wir uns doch noch genauer ansehen, wie Sie darauf kommen.

M.: Ich weiss es einfach. Was gibt es da noch zu reden, sagen Sie einfach gar nichts mehr, lassen Sie mich in Ruhe!

T.: Hmmh, und dann? ...Ich weiss im Moment nicht gut, ob ich Sie jetzt besser in Ruhe lassen soll, so wie Sie es wollen, oder doch weiterfragen. Ich will einerseits Ihre Grenzen respektieren aber ich finde andererseits Ihre Beschwerde auch so wichtig. Schliesslich geht es Ihnen darum, ob ich überhaupt mit Ihnen Therapie machen will.

(M. schweigt wieder)

Mentalisierungskonzept und Psychoanalyse 
T.: $\quad$..und ich weiss immer noch nicht, warum ich so unwillig auf Sie wirke. Vielleicht übersehe ich etwas. Habe ich irgendetwas gesagt, getan oder gelassen, dass Sie so gegen mich aufbringt? ... Aber um das zu klären, müssten wir weiter machen... .

M.: Ja, Sie haben etwas gemacht, und das tun Sie immer nur bei mir. Sie gucken immer so ärgerlich zu mir herüber. Sie wollen nicht mit mir arbeiten, das liegt doch auf der Hand... .

T.: Halt, halt, halt, nicht so schnell. Das sind so viele Themen auf einmal, ich komme gar nicht mit. Also bitte für mich noch einmal langsam, OK? ... Ich sehe Sie so ärgerlich an, und zwar immer, richtig?

M.: Ja.

T.: Und wie kommen Sie darauf, dass ich dann immer ärgerlich bin?

M.: Das kann ich Ihnen sagen: Sie haben echt solche Falten auf der Stirn, wenn ich etwas sage und Sie mich dann ansehen.

T.: Hmmh, ach so kommen Sie darauf. Sie geben sich alle Mühe, gut mitzumachen, und dann müssen Sie jedes Mal erleben, wie ich Stirnfalten bekomme, wenn Sie reden. So habe ich die Sache vorher noch nicht gesehen, und jetzt beginne ich langsam zu verstehen, warum Sie so wütend auf mich sind. Eine schwierige Situation zwischen uns, aber ich bin doch froh, dass Sie weiter mit mir an der Klärung arbeiten. Denn solche Probleme wie jetzt diese zwischen uns erleben Sie ja oft in Ihrem Alltag, und dann entsteht immer ein Scherbenhaufen. Deshalb haben wir ja auch abgesprochen, dass wir genau untersuchen, wenn so etwas auch in der Therapie geschieht. Aber ich bin mir nicht sicher, ob das auch in Ihrem Sinne ist ...

M.: Ja, das ist es. Endlich kann ich Ihnen einmal so richtig sagen, was mir nicht passt und Sie hören mir zu. Ja, das ist gut.

T.: Gut, dann würde ich gerne noch etwas wissen: Dass Sie wütend sind, weil ich ärgerlich aussehe, habe ich jetzt verstanden, aber noch nicht, warum Sie denken, dass ich nicht mit Ihnen arbeiten will. Woher wissen Sie das so genau? 
M.: Na, wenn Sie sich über mich ärgern, dann ist doch klar, dass Sie mich loswerden wollen.

T.: Wieso ist das so klar? ...Hmmh, es kostet mich in der Tat sehr viel Konzentration und Kraft, um Ihren psychologischen Theorien zu folgen und mir gleichzeitig vorzustellen, wie schlecht es Ihnen hinter Ihrer Fassade gehen könnte. Das könnte auch meine Stirnfalten erklären. Und sicher werden wir auch das Ärger-Thema noch genauer verstehen. ... Aber Sie wirklich loswerden wollen geht doch viel weiter! Was habe ich getan, dass Sie das von mir denken?

M.: Hmmh, weiss ich eigentlich nicht, ... vielleicht hat es auch gar nicht so sehr mit Ihnen zu tun....

T.: Wie meinen Sie das?

M.: Wissen Sie, ich brauche nur einen Ansatz von einer Stirnfalte zu sehen, dann muss ich sofort an meinen Vater denken. Na, Sie kennen ja die Geschichte.

T.: Ja, ich erinnere mich genau, das war in der Tat schrecklich für Sie. Und wie hat Sie das in unserem Gespräch beeinflusst? Was ist da genau in Ihnen vorgegangen?

Daraufhin beginnen wir, mehr von Frau M.s inneren Prozessen zu verstehen, und wie diese nach aussen sichtbar werden. Der weitere, weit weniger heftige und deutlich ruhigere Gesprächsverlauf ähnelt von aussen betrachtet eher einer Therapie mit einer neurotisch strukturierten Patienten. Das unmittelbare Erleben unserer Interaktion hat einen beruhigenden und motivierenden Effekt. Dies ist die Vorbereitung auf das spätere Hinterfragen ihrer Gewissheit, dass ich sie nicht behandeln wolle. Kurz vor Ende der Sitzung tut sie diesen Schritt schliesslich spontan selbst.

An dieser Sequenz wird deutlich, wie kleinschrittig, bewusstseinsnah und alltagssprachlich Frau M. und ich in der MBT das intersubjektive Feld erforschen und gemeinsam Bedeutung konstruieren. Etliche nicht genannte Aspekte hätten wir genauso gut ansprechen können, jedoch geht es in diesem Dialog nicht so sehr um den Inhalt der intrapsychischen und sprachlichen Vorgänge, sondern vor allem darum, ob und wie der Prozess gelingt, dass Frau M. zu einem mentalisierten Bild von sich und ihren Gefühlen, von mir und von unserer Interaktion kommt. Das zu untersuchende Feld ist nicht die erinnerte Vergangenheit mit ihrem Vater sondern 
die übertragungsbedingte Beeinflussung unserer Mentalisierungsfähigkeit und Interaktion im Hier und Jetzt.

\section{$4 \quad$ Fazit}

Selbst schwer strukturell gestörte Patienten können im Rahmen mentalisierungsfördernder Dialoge ohne ein einziges therapeutisches Interpretationsangebot unbewusster Inhalte zu wichtigen Einsichten in ihre Übertragung kommen. Dass sie es selbst entwickeln und formulieren können, macht sie stolz und motiviert. Es stärkt ihr Erleben von Selbstkohärenz und Selbstwirksamkeit, und es freut den Therapeuten. Damit dieser Prozess gelingt, muss sich der Therapeut von allem verabschieden, was eine Mentalisierungsbremse darstellen könnte. Er muss aktiv und unmittelbar erfahrbar seine Ich-Funktionen zur Verfügung stellen, besonders seine eigene Mentalisierungsfähigkeit und selektiv seine Gegenübertragung, ohne jedoch dem Patienten seine Gefühle oder Sichtweise aufzudrängen.

Wenn die Patienten ihre Mentalisierungsfähigkeit selbst in angespannten Zuständen hinreichend aufrecht erhalten oder schnell wiederherstellen können, wird es Zeit für eine andere, mehr interpretative Interventionstechnik, wie wir sie bei neurotisch strukturierten Patienten anwenden - dann hat die MBT ihren Zweck erfüllt.

\section{$5 \quad$ Literatur}

Bateman, Anthony und Fonagy, Peter (1999): Effectiveness of Partial Hospitalization in the Treatment of Borderline Personality Disorder: A Randomized Controlled Trial. In: American Journal of Psychiatry, Heft 156, 1563-1569.

Bateman, Anthony und Fonagy, Peter (2001): Treatment of Borderline Personality Disorder with Psychoanalytically Oriented Partial Hospitalization: An 18-Month Follow-up. In: American Journal of Psychiatry, Heft 158, 36-42.

Bateman, Anthony und Fonagy, Peter (2003): Health Service Utilisation Costs for Borderline Personality Disorder Patients Treated with Psychoanalytically Oriented Partial Hospitalisation versus General Psychiatric Care. In: American Journal of Psychiatry, Heft 160, 169-171.

Bateman, Anthony und Fonagy, Peter (2004): Psychotherapy for Borderline Personality Disorder.Mentalization-Based Treatment. Oxford, NewYork: Oxford University Press. (Deutsche Übersetzung: Psychotherapie der BorderlinePersönlichkeitsstörung. Ein mentalisierungsgestütztes Behandlungskonzept. Giessen: Psychosozial-Verlag, 2008). 
Bateman, Anthony und Fonagy, Peter (2006): Mentalization-Based Treatment for Borderline Personality Disorder. A Practical Guide. Oxford, NewYork: Oxford University Press.

Bateman, Anthony und Fonagy, Peter (2008): 8-Year Follow-up of Patients Treated for Borderline Personality Disorder: Mentalization-Based Treatment versus Treatment as Usual. In: American Journal of Psychiatry, Heft 165 (5), 631-638.

Bateman, Anthony und Fonagy, Peter (2009). Randomized Controlled Trial of Outpatient Mentalization-Based Treatment versus Structured Clinical Management for Borderline Personality Disorder. In: American Journal of Psychiatry, Heft 166, 1355-1364.

Bolm, Thomas (2009a): Mentalisierungsbasierte Therapie (MBT) als Gruppenpsychotherapie. In: Persönlichkeitsstörungen, Heft 13, 94-103.

Bolm, Thomas (2009b): Mentalisierungsbasierte Therapie (MBT) für BorderlinePersönlichkeitsstörung und chronifizierte Traumafolgen. Köln: Deutscher Ärzteverlag.

Bolm, Thomas und Herzog, Thomas (2009): Mentalisierungsbasierte Behandlung schwerer Borderline-Persönlichkeitsstörungen und Traumafolgen in der Klinik für Psychosomatische Medizin und Fachpsychotherapie, Christophsbad Göppingen. In: Birger Dulz, Philip Martius und Egon Fabian, Hrsg., Stationäre Psychotherapie der Borderline-Störungen in Deutschland. Stuttgart: Schattauer, 91-98.

Bolm, Thomas (2010a): Mentalisierungsdefizite und ihre Behandlung bei verschiedenen psychischen Störungen: What works for whom? In: Psychodynamische Psychotherapie, Heft 94, 205-215.

Bolm, Thomas (2010b). Mentalisierungsförderung. Wie viel Bindungserleben hilft welchem Patienten weiter? In: Psychotherapeut, Heft 55, 335-338.

Fonagy, Peter; Jurist, Elliot; Gergely, Giörgy; Target, Mary (2002): Affect Regulation, Mentalization and the Development of the Self. New York: Other Press. (Deutsche Übersetzung: Affektregulierung, Mentalisierung und die Entwicklung des Selbst. Stuttgart: Klett-Cotta, 2004).

Lindner,Wulf-Volker (2005): Das Göttinger Modell der Anwendung der Psychoanalyse in Gruppen: Geschichte, Theorie, therapeutische Konzepte und Anwendung im sozialen Feld. In: Gruppenpsychotherapie und Gruppendynamik, Heft 41, 99-129.

Mertens, Wolfgang (1993): Einführung in die psychoanalytische Therapie. Bd.2, 2. Aufl. Stuttgart: Kohlhammer. 
Nolte, Tobias; Hudac, Caitlin; Mayes, Linda C.; Fonagy, Peter., Blatt, Sydney J., Pelphrey, Kevin (2010): The Effect of Attachment-Related Stress on the Capacity to Mentalize: An Fmri Investigation of the Biobehavioral Switch Model. In: Journal of the American Psychoanalytic Association, Heft 58, 566-573.

Streeck, Ulrich und Leichsenring, Falk (2009): Handbuch psychoanalytisch-interaktionelle Therapie. Behandlung von Patienten mit strukturellen Störungen und schweren Persönlichkeitsstörungen. Göttingen: Vandenhoek\&Ruprecht. 